\title{
Can GPs diagnose benign paroxysmal positional vertigo and does the Epley manoeuvre work in primary care?
}

Benign paroxysmal positional vertigo (BPPV) in adults is a common cause of dizziness seen in general practice with a 1year prevalence of $1.6 \% .^{1}$ It is characterised by brief episodes of dizziness or vertigo typically triggered by rapid changes in the position of the head and can be associated with nausea which may persist. ${ }^{2}$ BPPV can resolve spontaneously within weeks or months. ${ }^{2}$ It can present in clusters and can recur after remission. ${ }^{2}$ This short paper is based on a critical literature review.

\section{DIAGNOSING BPPV}

GPs can confirm a diagnosis of BPPV using the Dix-Hallpike test. ${ }^{2,3}$ The patient is moved quickly 'from a sitting position to lying with the head tipped $45^{\circ}$ below the horizontal, $45^{\circ}$ to the side, and with the side of the affected ear (and semicircular canal) downwards.' ${ }^{2}$ The Dix-Hallpike test is positive when torsional (rotatory) nystagmus occurs when the head is turned to the affected ear. ${ }^{4}$ In a prospective study of diagnosis of vertigo in general practice, a positive Dix-Hallpike test had a positive predictive value of $83.3 \%$ and a negative predictive value of $52 \%$ in diagnosing BPPV. ${ }^{3}$ Having done so, GPs can then usually resolve the condition through a manipulation called the Epley manoeuvre.

Figure 1. The Epley manoeuvre for treating benign paroxysmal positional vertigo.

This article was published in Otolaryngology - Head and Neck Surgery, 107(3), Epley JM, The canalith repositioning procedure: for treatment of benign paroxysmal positional vertigo, 399-404, Copyright Elsevier 1992.
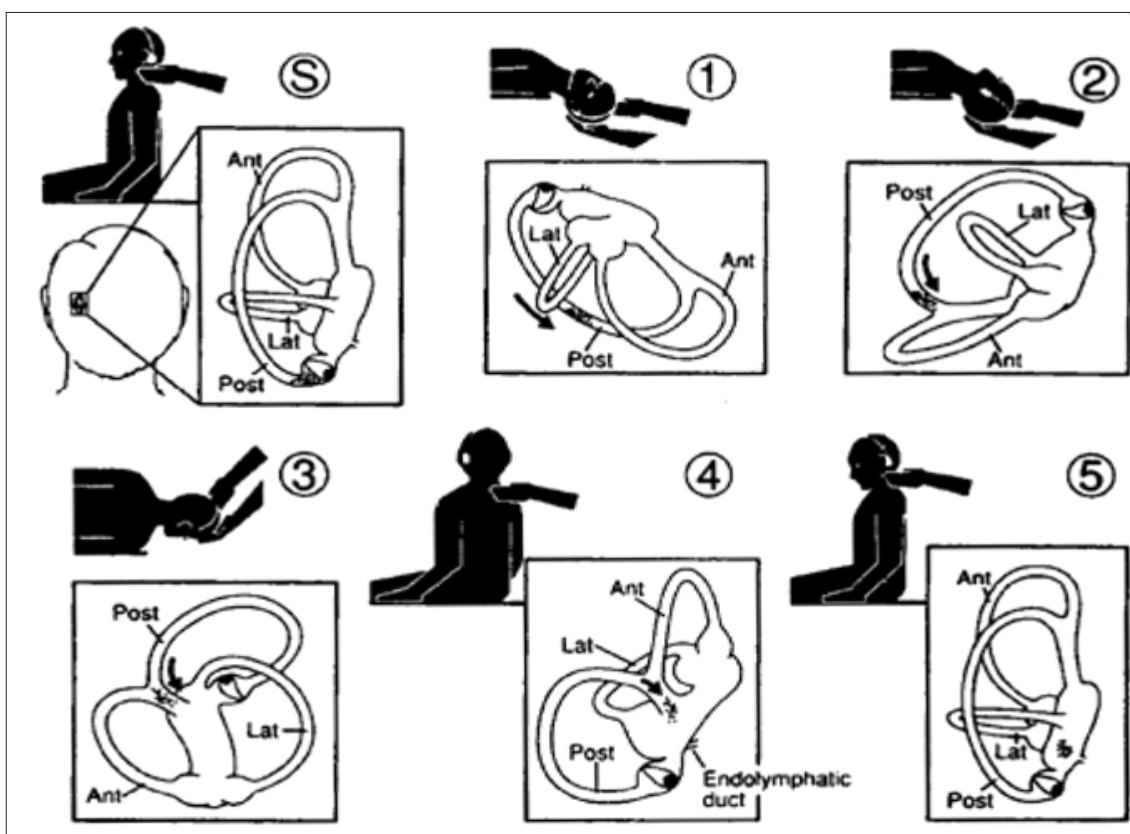

Figure 1. Positions for canalith repositioning procedure targeting left posterior semicircular canal (PSC). Solid figures = side view. Boxes = operators exposed view of the labyrinth, showing gravitating canaliths. Semicirvular canals are labeled. S(start) Patient is seated, operator behind, oscillator is applied. (1) Head is placed over end of table, $45^{\circ}$ to the left (canaliths gravitate to centre of PSC). (2) While head is kept tilted downward, it is rotated $45^{\circ}$ right (canaliths reach common crus). (3) Head and body are rotated until facing down $135^{\circ}$ from supine position (canaliths traverse common crus). (4) While head is kept turned right, patient is brought to sitting (canaliths enter utricle). (5) Head is turned forward, chin is tilted down $20^{\circ}$. Generally, pause at each position until vertigo and nystagmus stop. Make sure the nystagmus beats in the same direction during each step as this conforms movement of the debris in the desired direction. Keep repeating entire sequence ( 1 to 5 ) until no nystagmus in any position.

\section{EPLEY MANOEUVRE}

The Epley (canalith repositioning) manoeuvre is a 'safe and effective treatment' for BPPV. ${ }^{2}$ It consists of 'a series of four quick movements of the head and body from sitting to lying, rolling over, and back to sitting (Figure 1). Each position is maintained until positional nystagmus has disappeared, indicating cessation of endolymph flow'. ${ }^{4}$ The Epley manoeuvre has been shown to be beneficial after one session when $77 \%$ of patients reported effective relief and an additional $20 \%$ of patients reported the same the following week after the second session. ${ }^{4}$ Patients are advised to perform self-treatment at home after receiving the Epley manoeuvre. ${ }^{4}$

\section{IS IT FEASIBLE TO IMPLEMENT THE EPLEY MANOEUVRE IN GENERAL PRACTICE?}

The Epley manoeuvre in general practice produces similar results when implemented in secondary or tertiary centres..$^{5}$ A randomised, prospective, double-blind, sham-controlled study determined whether the Epley manoeuvre is effective for treating BPPV in primary care. ${ }^{5}$ At baseline the intervention group received the Epley manoeuvre and the control group received a sham manoeuvre which consisted of the Epley manoeuvre performed on the unaffected side. ${ }^{5}$ At 1 week and 2 weeks both groups received the Epley manoeuvre. ${ }^{6}$ Initial improvement was statistically significant, as after the first treatment $34.2 \%$ of patients in the intervention group had a negative DixHallpike test, compared with $14.6 \%$ in the control group $(P$ value $=0.04 ; 95 \% \mathrm{Cl}=$ 1.03 to 5.33$).{ }^{5}$ This study concluded that the number of patients who were successfully treated with the first Epley manoeuvre was statistically significant compared to the control group, and that GPs could use the Epley manoeuvre to treat BPPV. ${ }^{5}$ 


\section{Liberating the NHS}

Glasziou suggested that the Epley manoeuvre has been slow to be implemented into primary care because of the level of skill involved and a lack of confidence with the Dix-Hallpike test and the Epley manoeuvre. ${ }^{1}$ This can be addressed with training; for example, using a video showing the Dix-Hallpike test and Epley manoeuvre. ${ }^{1}$ It is useful to have another member of staff to assist when carrying out the test and the manoeuvre. The staffing implications need to be considered.

In a 10-minute consultation, a GP could take a history and perform Rinne's and Weber's tests followed by the Dix-Hallpike test and the Epley manoeuvre.

\section{CONCLUSION}

The evidence suggests that BPPV can be diagnosed and subsequently treated with the Epley manoeuvre in general practice with great effect, thus reducing referrals to specialist centres. If the patient subsequently presents with unresolved symptoms they should then be referred. Further research needs to be undertaken to measure the effectiveness of the Epley manoeuvre in general practice through further randomised controlled trials. Avoiding long-term medication, and the consequent side effects, is another aspect of the cost-effectiveness of the manoeuvre.

The research evidence suggests this diagnostic manoeuvre and manipulation can be readily and successfully adopted in primary care.

\section{Sarah Cranfield, lan Mackenzie, and Mark Gabbay}

\section{REFERENCES}

1. Glasziou P, Heneghan C. Epley and the slow boat from research to practice. Evid Based Med 2008; 13: 34-35.

2. Hilton M, Pinder D. The Epley (canalith repositioning) manoeuvre for benign paroxysmal positional vertigo. Cochrane Database Syst Rev 2004; 2: CD003162.

3. Hanley K, O' Dowd T. Symptoms of vertigo in general practice: a prospective study of diagnosis. Br J Gen Pract 2002; 52: 809-812.

4. Lempert T, Gresty MA, Bronstein AM. Fortnightly Review: Benign positional vertigo: recognition and treatment. BMJ 1995; 311: 489-491.

5. Munoz JE, Miklea JT, Howard M, et al. Canalith repositioning maneuver for benign paroxysmal positional vertigo Randomized controlled trial in family practice. Can Fam Physician 2007; 53: 1048-1053.

DOI: 10.3399/bjgp10X515557
The new health White Paper proclaims, in its subtitle, the goal of 'liberating the NHS', but the ascendancy of the concept of 'wellbeing' threatens to consolidate the tyranny of health over patients and professionals alike. ${ }^{1,2}$

Having suggested before the election that the abolition of PCTs would be a 'promising way of saving money and improving primary care at a stroke', ${ }^{3}$ I find myself in an unfamiliar position of alignment with one of the more controversial proposals of the coalition government's White Paper. On first reading this hastily produced blueprint for drastic restructuring of the health service, I was struck by the claim that the proposed replacement for PCTs - local GP consortia - would 'increase efficiency by enabling GPs to strip out activities that do not have appreciable benefits for patients' health or healthcare'. But my excitement at the prospect of 'stripping out' all the sort of 'health promotion' and 'disease prevention' activities that have such a baneful effect on the health of our patients - starting with the NHS Health Check - was short-lived. These are exactly the sorts of activities that the Equity and Excellence White Paper, whose very title and every page indicate a spirit of continuity with the buzzwords and rhetoric of New Labour ('transparency', 'world-class', even 'information revolution'), is determined to pursue, indeed, to enforce on general practice.

The familiar weasel words of 'choice', 'competition', and 'empowerment' thinly disguise compulsion and coercion. The proposed consortia will be assembled by a process of forced collectivisation: GPs will have no choice about the terms on which we compete in the new primary care market. We will also have a 'duty to participate' with local government authorities, who will be given major new powers, including taking over many of the functions of the old PCTs. In particular, we will be obliged to surrender to the 'strategic role' of the proposed local authority 'health and wellbeing boards'. (To adapt an old adage, 'Those who can do, those who can't, take on a strategic role'.) These boards will provide a new base from which the zealots of public health can promote their moral crusades (from 'safe sex' to 'five a day') and hype up public anxieties with their imaginary epidemics and pandemics.

The expansion of health reflected in the relatively new, but now universally promoted, coupling of 'health and wellbeing', has major consequences for society. Instead of being regarded as the absence of disease, the default state of robust citizens in a mature democracy, health has become the transcendent goal of the fragile and vulnerable individuals of the risk society. Wellbeing - like its close relation, 'happiness', another policy 'outcome' for both Tony's 'Third Way' and Dave's 'Big Society' - can only be attained through the pursuit of an ascetic lifestyle and regular submission to medical surveillance. The problem for the NHS is that the burden of demand resulting from the healthrelated anxieties and expectations that are unleashed by this process are unsustainable. The reforms proposed in the new White Paper, like its predecessors, will merely ensure that more and more people, falling short in achieving the desired and promised outcomes of health and wellbeing, will feel ill, fostering spiralling costs in both primary and secondary health care.

\section{REFERENCES}

1. Department of Health. Equity and excellence: Liberating the NHS. White Paper. July 2010. http://www.dh.gov.uk/prod_consum_dh/groups/ dh_digitalassets/@dh/@en/@ps/documents/digit alasset/dh_117794.pdf (accessed 6 Aug 2010).

2. Fitzpatrick, M. The tyranny of health: doctors and the regulation of lifestyle. London: Routledge, 2000.

3. Fitzpatrick, M. How to cut NHS spending and improve the health of the nation. Br J Gen Pract 2010; 60:(572): 223.

DOI: 10.3399/bjgp10X515566 